\title{
LA INFLUENCIA DEL SISTEMA NEOLIBERAL EN LAS ELECCIONES OCUPACIONALES Y EL IMPACTO EN LA SALUD Y BIENESTAR DESDE UNA PERSPECTIVA CUALITATIVA
}

\begin{abstract}
THE INFLUENCE OF THE NEOLIBERAL SYSTEM IN THE OCCUPATIONAL ELECTIONS AND ITS IMPACT ON HEALTH AND WELLBEING, FROM A QUALITATIVE PERSPECTIVE.
\end{abstract}

\author{
Samanta Rolack Jaramillo ${ }^{1}$, Vanessa Triviños Rubilar ${ }^{2}$, Paulina Troncoso Henriquez ${ }^{3}$
}

\section{RESUMEN}

El objetivo del artículo es presentar el proceso de investigación, análisis, resultados y discusión respecto a la influencia del sistema neoliberal en las elecciones ocupacionales y su impacto en la salud y bienestar en el ser humano desde una perspectiva cualitativa. Este estudio fue realizado con una muestra no representativa de población económicamente activa de la ciudad de Valdivia, Chile, en base a la metodología de producciones narrativas. Los principales resultados obtenidos es la constatación de una serie de factores interrelacionados que influyen en las elecciones ocupacionales, considerando elementos propios del sistema neoliberal como uno de los factores influyentes al momento de elegir una actividad productiva, carrera a estudiar, pasatiempos y otros, lo que a su vez impacta en la configuración de la participación ocupacional en las áreas de desempeño ocupacional. Así finalmente se cuestiona el concepto de elecciones ocupacionales, si realmente se dan de forma autónoma y libre, o si debemos reformular este concepto, considerando los factores que influyen en tal proceso y teniendo en cuenta al ser humano como un ser ocupacional, actor social, y junto con ello, la práctica de la Terapia Ocupacional.

\section{Palabras claves:}

Sistema neoliberal, elecciones ocupacionales, salud, bienestar, terapia ocupacional.

\footnotetext{
${ }^{1}$ Terapeuta Ocupacional, Licenciada en Ciencias de la Ocupación Humana Universidad Austral de Chile, Terapeuta Ocupacional de la Sala de Rehabilitación Basada en la Comunidad y del Centro Diurno El Chañar en la ciudad de Copiapó, contacto: e-mail samantarolackj@gmail.com.

${ }^{2}$ Terapeuta Ocupacional, Licenciada en Ciencias de la Ocupación Humana Universidad Austral de Chile, Terapeuta Ocupacional de Centro de Estimulación Temprana Incapoyen y Programa SENDA, contacto: vanessatrivinosrubilar@gmail.com.

${ }^{3}$ Terapeuta Ocupacional, Licenciada en Ciencias de la Ocupación Humana, Universidad Austral de Chile, Terapeuta Ocupacional de COSAM de Hualpén, contacto: pauli.thz@gmail.com.
} 


\begin{abstract}
The purpose of this article is to show the process of investigation, analysis, results and discussion about the influence of the neoliberal system in the occupational elections and its impact on health and wellbeing from a qualitative perspective. This study was made with a non-representative sample of the active economic population of the city of Valdivia, Chile, with the methodology of narrative productions. The principal results obtained are the verification of a series of interrelated factors that influence the occupational elections, considering elements of the neoliberal system as one of the influential factors at the moment of choosing a productive activity, a university degree, hobbies and others, which at the same time hits the configuration of the occupational participation in the areas of occupational performance. Finally the concept of occupational elections must be questioned, if they're really stand-alone and exempt choices, or if we must rethink its definition, given the factors that influence this process, and considering the human as an occupational being, as a social actor, and with it the practices of Occupational Therapy.
\end{abstract}

\title{
Key words:
}

Neoliberal system, occupational elections, health, wellbeing, occupational therapy. 


\section{INTRODUCCIÓN}

Numerosos son los autores que realizan una crítica desde la sociología, economía y otras disciplinas, de los aspectos negativos del sistema neoliberal al coartar la libertad de acción de los individuos.( Angelcos, Pérez, Sémbler, 2006; Boron, 2008; Chomsky, 2004; Fernandez, 1990; Fromm, 1987; Gandarilla, 2005; Max Neef, 1993; Bourdieu, 2003; Mondaca, s.f., Rifkin, 2000; Soros, 1999), sin embargo, es insuficiente la información desde las ciencias que analizan, reflexionan y teorizan sobre y desde la ocupación humana, es decir, las Ciencias de la Ocupación, la cual considera la expresión del ser humano a través de la ocupación. (Wilcock, 1998). La ocupación, nos mantiene en la realidad, en el día a día, nos ofrece una fuente de salud, al existir situaciones donde se ausente o se altere el significado de esta, el ser humano puede ver amenazado su salud y bienestar (Talavera, 2004).

Sin embargo, desde las ciencias de la ocupación, autores como Yerxa (1933, citada en Kronenberg \& Polard, 2007) y Wilcock (1993 citada en Kronenberg \& Polard, 2007 p. 83) señalan: "los terapeutas ocupacionales consideran a los seres humanos como seres ocupacionales". Otros autores agregan que "tener una ocupación digna y que otorgue significado a la existencia de la persona es tan fundamental para la salud y el bienestar como comer, beber, y ser amado" (Kronenberg, 1999, Mason 2002, Townsed,2004, citados en Kronenberg \& Polard, 2007) a su vez, Wilcock (1998) menciona que el desarrollo ocupacional ocurre en paralelo al desarrollo biológico y cultural, siendo producto de la interacción de estos conceptos.

Estos autores constituyen la base que nos permite cuestionarnos acerca de la interrelación entre el contexto social, económico, político y su relación con las elecciones ocupacionales que pueda realizar el ser humano, ya sea en el desarrollo de una ocupación en particular, o varias en general. Desde nuestra disciplina, existen escasas investigaciones acerca del análisis de cómo los seres ocupacionales se ven influenciados por el contexto político-económico-sociológico al momento de realizar elecciones ocupacionales, y el respectivo impacto de estas en la salud y bienestar.

Prudant (2014) establece un análisis desde la perspectiva de salud mental y la influencia de la biopolítica del desarrollo, postulando que el cambio que ha tenido este último concepto, actualmente neoliberalismo, puede ser entendido desde una doble perspectiva. Desde los efectos concretos en prácticas clínicas analizando las dimensiones de la vida que se ven afectadas por el entorno que se forma tras la aplicación de políticas y prácticas neoliberales, traducido a la aparición de trastornos mentales o bien en la pérdida de bienestar humano. En conjunto con lo anterior políticas generadas desde el neoliberalismo contribuyen a la desigualdad social, y transformaciones sufridas en la regulación laboral, tales como la precariedad laboral en la era de globalización. 
Es por ello que el objetivo de la presente investigación es responder, desde una perspectiva cualitativa, la siguiente interrogante: ¿es el sistema neoliberal un factor que influye en las elecciones ocupacionales? ¿Cuál es el impacto en la salud y bienestar? Para ello, se trabajó con adultos económicamente activos de la ciudad de Valdivia, con el objetivo de analizar la configuración de sus elecciones ocupacionales según lo esperado en su etapa evolutiva, donde se espera que sean económicamente independientes de terceros, lo cual permite realizar una mayor gama de elecciones (Zacarés y Serra, 1996).

Para fines de la investigación, definiremos a continuación los conceptos de sistema neoliberal y elecciones ocupacionales en base a la revisión bibliografía realizada en la presente investigación; entendiendo que son conceptos complejos de definir por su base histórica e ideológica, sin embargo, es necesario consensuar su definición para cumplir los fines investigativos:

Se entenderá como sistema neoliberal como aquella forma de organización sociopolítico-económico-administrativa dominado por el mercado como principio ordenador, donde la acción del estado es subsidiaria y restringida, existiendo desigualdades estructurales y concentración de riquezas en sectores (Garreton, 2012, Max-Neef, Angelcos et al, 2006, Hachete, citado en Angelcos et al, 2006, Mondaca, s.f, García, 2010, Rifkin, 2000, Chomsky, 2004, Boron, 2008)

Se entenderá como elecciones ocupacionales, como aquellas decisiones tomadas por el ser ocupacional respecto a lo que se desea hacer y cómo hacerlo, de forma libre y voluntaria, ya sea iniciando o terminando actividades, alrededor de nuestro presente o nuestro futuro; pueden ser decisiones que se toman diariamente y con impacto inmediato, o decisiones más importantes que implican un compromiso para sostener la acción a largo plazo, produciendo un impacto fundamental en la vida, construyéndola en relación con sus roles, modificando hábitos, en base a un contexto personal, historia de vida, intereses y motivaciones, basándonos en lo señalado por Kielhofner (2004), sin embargo no consideraremos la diferencia que este autor realiza con las elecciones de ocupaciones y actividades, ya que precisamos de un concepto que englobe las mayorías de las decisiones. Estas elecciones de participar en diferentes ocupaciones, se agrupan según al área ocupacional que pertenecen: productiva- autocuidado y ocio y tiempo libre. (DNHW \& CAOT, 1983)

En base a lo anterior, cabe preguntarnos de qué forma se plasma en las narrativas de las personas la influencia del sistema neoliberal entiendo a este como un contexto de desempeño, en las elecciones ocupacionales, y si esto incide en su salud y bienestar. Incluso diversos autores como Granados (2002), Marrero (2007) y Rodríguez (2000) señalan que este sistema propicia que los seres humanos vean influenciada de forma negativa su salud y bienestar, por lo que resultan cuestionamientos importantes que invitan a iniciar un proceso de investigación y análisis desde las ciencias de la ocupación.

\section{MARCO METODOLÓGICO}


Este es un estudio exploratorio, de corte transversal, con una muestra no estadística ni probabilística utilizando el enfoque cualitativo, a través de las producciones narrativas (Balasch y Montenegro, 2003). Estas consisten en textualizar, de forma organizada y sistematizada, las ideas surgidas en las conversaciones mantenidas con las y los participantes, con la finalidad de crear un relato con lógica argumentativa e interpretativa. Esta lógica se genera a través de la visión situada del investigador en un tiempo y contexto determinado, en el que se comparte un lenguaje común, haciendo posible la producción de un conocimiento situado en este entramado relacional. Hay que tener en cuenta que esta metodología trata el lenguaje desde una perspectiva dialógica, es decir, como un flujo de acciones, una actividad que está en constante construcción y reconstrucción en las diferentes interacciones donde se reproducen significados gracias a su carácter responsivo (Balasch y Montenegro, 2003 p. 46).

Una vez realizado el texto, se revisa y se presenta a las/los participantes para que lo revisen, comenten, modifiquen y otorguen su autorización para que la información pueda ser utilizada en el estudio; obteniendo así un texto definitivo. Estos textos no serán presentados como datos o registros discursivos a analizar, sino que con ellos se pretende proporcionar una visión del fenómeno. (Balasch y Montenegro, 2003).

Se realizaron cinco producciones narrativas a los siguientes participantes:

* J. I. D., de profesión enfermera de 29 años, con la cual se realizaron 2 sesiones.

* A.V., Empresario independiente y estudiante de 37 años, con el cual se realizaron 2 sesiones.

* C.Q. Asistente Social de 36 años, con la cual se realizaron 4 sesiones.

* M.T, trabajador dependiente y músico de 35 años, con el cual se realizaron 3 sesiones.

* V. G. Egresado de antropología de 29 años, con el cual se realizaron 2 sesiones.

La producción del texto ha sido generada de la siguiente forma:

* Se realizó inducción sobre el tema de estudio, junto con mostrar la metodología y presentar los consentimientos informados con la invitación a participar de la investigación.

* Una vez firmados los consentimientos informados, se realizaron sesiones de conversación sobre el tema de estudio

* Se realizó la textualización de estas conversaciones en un documento que contiene dichas conversaciones y resume la postura del participante respecto al tema de estudio.

* Los participantes revisaron las textualizaciones y realizaron sus modificaciones para ajustarlo a su punto de vista. 
* Finalmente los investigadores realizarán un análisis en base a las textualizaciones obtenidas y posteriormente se extraerán las conclusiones relacionadas a la pregunta de investigación.

Los participantes han sido seleccionados bajo los siguientes criterios:

* Disponibilidad de tiempo para participar en los diálogos correspondientes de la investigación.

* Grado de cercanía o familiaridad con los investigadores, para facilitar el abordaje de los temas.

* Interés respecto al tema a analizar

\section{ANÁLISIS Y RESULTADOS}

Se han estructurado bajo tres ejes principales:

\section{Neoliberalismo: su definición y características.}

Los participantes coinciden con lo señalado por los autores críticos al neoliberalismo, sobre su aspecto inequitativo y sus efectos negativos en la calidad de vida de las personas. (Pusey 2003; Saunders 2002; Chomsky 2004). Destacan las brechas acentuadas de desigualdad, beneficiando a un sector minoritario de la población, como por ejemplo en el ámbito de la salud, donde aquellos que poseen menos recursos económicos se ven desplazados, existiendo normalización de estas situaciones de desigualdad, como una estrategia para mantener y sostener el modelo neoliberal.

Señalan su aspecto competitivo, evaluado por algunos como positivo en relación a ser un incentivo a mejorar el desempeño laboral, lo que a su vez trae consecuencias como que la persona se enferme por ellos manifestándose a través del estrés, depresión y desgaste en las relaciones sociales.

Destacan la monopolización y la explotación de los recursos, lo que impacta finalmente en el acceso a los productos que los participantes adquieren, señalando que les gustaría que exista una mayor fiscalización y no el rol de espectador actual del estado; agregan la desconfianza y descontento con el actual sistema de pensiones y salud pública, enfatizando en la necesidad de un aspecto benefactor y no subsidiario del estado chileno, lo que coincide con lo mencionado por Cifuentes (2013, citado en Toro, 2013) donde se configura esta transición exigida desde los movimientos sociales del país y el rol del estado chileno en la economía nacional post-régimen militar. (Garreton, 2012, Max-Neef, 1993, Angelcos et al, 2006, Hachete, citado en Angelcos et al, 2006, Mondaca, s.f, García, 2010)

\section{II: Sobre el sistema neoliberal y su interrelación desde un análisis de las estructuras sociales, político-administrativas.}


En relación a lo anterior sobre el protagonismo del Estado y su rol subsidiario, se desprende la discriminación positiva hacia la población con menos recursos en desmedro de la clase media, (Barrere, s.f.), como ejes coyunturales de política pública donde los beneficios estatales recaen en la población con menos recursos, esta marginación se da por estrategias del sistema económico que resultan en la discriminación de sectores, existiendo dos vías que se contraponen entre sí como metodologías para lograr metas aspiracionales: la primera de ellas, son el esfuerzo y los talentos personales como estrategia de la meritocracia apoyada en el individualismo como vía de éxito, (Young, citado en García, 2006, p21), mientras que la segunda, recae en políticas subsidiarias estatales, donde el rol protagónico recae en un factor externo al individuo. Sin embargo los participantes que se identifican con la clase media, no se perciben como población objetivo de ayuda Estatal, por lo que cobra mayor relevancia el poseer lo necesario a nivel individual que permita un desenvolvimiento adecuado en la sociedad, sin factores externos que los auxilien.

Surge así la meritocracia (Young, citado en García, 2006, p21), como el medio para conseguir una buena calidad de vida, señalando el esfuerzo personal como vía de éxito, naturalizándolo para alcanzar el bienestar subjetivo; una de las frases textuales de los participantes refiere: "si no te subes al tren, te quedas abajo", esta analogía señala la importancia de sentirse incluidos en el sistema, y convertirse en objetos prescindibles para el sistema, por lo que se acentúa la competencia. Ello implica esforzarse por poseer lo necesario para un desenvolvimiento adecuado en la sociedad, bajo los criterios del modelo neoliberal en relación a lo que señala Bordieu (2003) sobre el volumen en el espacio social, lo cual se ahondara más adelante a medida que se describan los resultados.

Señalan que hay una relación directamente proporcional entre los conceptos de poder y dinero, explicitando que al tener capital económico aumenta el estatus, el cual puede ser usado de forma positiva o negativa sobre otros, pues tiene relación con el acceso a bienes y conocimientos, sin embargo agregan que es un complemento al valor de la persona en cómo es con su entorno o como profesional.

Los participantes caracterizan a la sociedad como materialista y aspiracional, considerándola como discriminatoria, individualista y competitiva, en la cual se desarticulan las redes de confianza, estableciéndose un vínculo ganancial, perdiendo la expectativa humana, como la solidaridad, convirtiendo a las personas en objetos, (Fromm, 1987; Pusey 2003; Saunders 2002; Rifkin, 2000), lo que es visualizado de forma negativa por los participantes, pues le resultan significativos los valores altruistas como la confianza, solidaridad y el compartir en comunidad. Sin embargo, se observa un discurso ambivalente pues no se correlaciona con lo que se ejecuta, ya que al realizar las elecciones ocupacionales, prima el individualismo sobre el sentido colectivo, para no ser marginado del sistema y obtener poder en el espacio social. (Bordieu, 2003)

Los participantes identifican como los principales efectos que genera el sistema económico en la sociedad el de aturdidor, buscador de pasividad de la población y limitante en sí mismo por no permitir vislumbrar una vía distinta a este, junto con la normalización del endeudamiento y problemas de salud asociados como depresión, estrés y sintomatología física asociada a ello (Behm, 2011; Fernández, 1998). 


\section{Elecciones Ocupacionales}

Los participantes realizan elecciones respecto a los servicios de salud, educación y otros, basados en la oferta propuesta por el mercado. Éstos corresponden a carácter público o privado, donde hay una clara diferencia entre ambos en cuanto a satisfacer sus demandas a corto plazo. Ahora entendiendo las elecciones ocupacionales como describimos al inicio, como decisiones con sentido y significado realizadas de una forma libre, es necesario realizar el análisis de que tan libre es escoger respecto a derechos básicos como salud y educación sien do que las opciones presentadas se encuentran enmarcadas dentro de un sistema económico. Somos seres ocupacionales, escoger en que dispositivo de salud quiero recibir mi atención se encuentra íntimamente relacionado con los roles e intereses, más aún si se trata de educación, considerando que es una piedra inicial para construirnos como persona.

Considerando que nuestros entrevistados refieren que su opción por el sistema privado se basa en la calidad y atención a sus demandas educación de calidad que les permita a sus hijos mayores herramientas para un futuro o atención de salud que pesquise a tiempo patologías y que las cubra de manera adecuada, (Boron, 2008), sin embargo enfatizan que les gustaría que el Estado tuviese un mayor protagonismo y que corrigiera esta situación; por consiguiente, se observa que surge la desconfianza en las instituciones sociales del estado por el limitado campo de acción que poseen y por la calidad deficiente entregada, consideran que han mejorado ciertos servicios tales como educación o salud, sin embargo no de forma suficiente, lo cual no resulta en un aumento de confianza hacia estos y por ende, no son elegidos. En esta misma dirección, al relacionar lo anterior con la proyección actual y a futuro, sobre el ejercicio de su rol como padre o madres, señalan que no desean que su hijos reciban servicios de mala calidad, por que repercutiría negativamente en la vida de estos, dado que no contarían con la calidad que sí entrega el servicio privado en el ámbito de educación por ejemplo, el cual brinda mayores herramientas que el sistema público, lo que le permitan una mejor posición en el espacio social (Bordieu, 2003). Sin embargo, en otras preguntas, manifiestan claramente la tendencia que le gustaría que dichos servicios fuesen entregados por el Estado como garante de esos servicios, que existiese un sistema público al cual todos pudiesen acceder para una mayor igualdad, por lo que se observa una ambivalencia en cómo les gustaría que fuesen los servicios y el sistema en general y entre lo que eligen finalmente.

Se considera la actividad productiva remunerada como la vía que permite la adquisición de capital económico y que este, a su vez, permite la adquisición de otros capitales (Bourdieu, 2003), siendo un factor relevante en cuanto al percibirse cohartado o incluido por el sistema; señalan que uno de los factores que influyen en la elección de una ocupación productiva, es la alta remuneración, que implica adquisición de capital objetivado (Bourdieu, 2003) donde el placer de realizar una ocupación es un elemento menos relevante en relación al poder económico-adquisitivo que esta entrega, evidenciándose la supeditación del poder económico sobre el placer entregado por una 
determinada ocupación, lo cual es descrito como una situación que favorece la aparición de un factor de riesgo ocupacional: la enajenación (Wilcock, 1998).

Al realizar la pregunta sobre qué sucedería si su actividad productiva se viera suprimida ante un despido, las participantes femeninas señalan esta situación como un evento caótico, teniendo que modificar de una forma considerable su estatus de vida, pues mediante esta actividad expresan valores altruistas y ven reflejada su vocación. Mientras que los participantes masculinos, señalan que buscarían otro empleo, sin expresar la relación vocación-actividad productiva, dando relevancia al ingreso económico que permite desarrollar una ocupación productiva.

En cuanto a las elecciones relacionadas con las áreas de autocuidado u ocio y tiempo libre, se observa la supeditación de estas por el área productiva; en cuanto a la duración de la jornada laboral, se coincide que disminuir las horas laborales entregaría mayor tiempo para estar con su familia o participar de actividades recreativas. En este sentido podemos decir que la relación de estos factores propicia un desequilibrio ocupacional (Wilcock, 1998).

En relación a su participación social, se observa que participan de instancias obligatorias de acuerdo sus roles, como agrupación de padres. Por otro lado participan de organizaciones sindicales, comunidad religiosa, club deportivo y otros de acuerdo a sus intereses (Kielhofner, 2004), sin embargo, esta participación se ve en desmedro en cuanto al tiempo destinado, en relación a la actividad productiva, observándose que las decisiones son desde una mirada individual y no colectiva. Debido a que la participación se ve condicionada de acuerdo a los beneficios personales que se obtienen al participar. Sobre la participación política, los factores que influyen en no participar de un partido son el considerarlos corruptos o no reflejar sus ideales, además de señalar el distanciamiento de lo que el colectivo social pide y lo que se representa finalmente. Se acusa a los políticos de no escuchar las demandas locales; esto no significa una limitante para identificar una tendencia política o para ejercer su derecho a votar.

Finalmente, se desprende que las elecciones ocupacionales son realizadas en base a una lógica aspiracional, relacionada con la acumulación de capital económico que permite obtener el resto de los capitales (Bordieu, 2003) lo cual se hace priorizando la actividad productiva por sobre actividades de ocio y tiempo libre, participación comunitaria y autocuidado. Esta lógica aspiracional tiene relación con la búsqueda del poder que permite posicionarse de una forma sobresaliente en el espacio social, sus poseedores se configuran como un grupo selecto bajo las relaciones dominantes - dominados (Bourdieu, 1983, citado en Meichsner, 2007).

Esta supeditación del área productiva en relación a las otras áreas ocupacionales, ocasiona una mayor acumulación de carga laboral, desplazando la participación en ocupaciones que generan placer por sí mismas correspondientes al área de ocioautocuidado, centrándose en el producto concreto que resulta en las ganancias que se obtenga de ello, tanto desde lo económico como los otros tipos de capitales, y lo que esto configura como poder en el espacio social. 


\section{Su impacto en la salud y bienestar.}

Los entrevistados coinciden que el bienestar es un estado relacionado con la propia percepción de sentirse y estar bien, satisfacción física como biológica, de necesidades personales y familiares y la armonía que existe en esos sistemas, sumado además a las dinámicas en el establecimiento de relaciones interpersonales, lo que coincide con el concepto de salud OMS (1948), entendido como el completo estado de bienestar físico, mental y social y no solamente la ausencia de afecciones o enfermedades. Junto a ello, desde las ciencias de la ocupación se agrega que tal concepto tiene relación con la libertad que pueda tener el individuo de participar en diferentes ocupaciones. (Townsed, E. \&Wilcock, A. 2002)

Respecto al cómo impacta el sistema neoliberal, de acuerdo a la forma en que lo definen y caracterizan y su influencia en las elecciones ocupacionales que realizan, los participantes señalan el impacto negativo en la salud y bienestar, donde están más propensos a adquirir patologías físicas y mentales producto de una alta carga laboral que se adquiere al tener que estar más tiempo participando de la actividad productiva, donde prima la competencia y el individualismo al momento de realizar una elección ocupacional. Con respecto a la influencia sobre adquirir bienes materiales en el bienestar subjetivo, señalan que sí hay un impacto sobre este último, sin embargo no es factor determinante de la felicidad, pero si un facilitador. Si bien entrega la posibilidad de acceder a la adquisición de bienes, como vehículos de transporte, libros, música, tecnología, entre otros, no garantiza un estado de felicidad para ellos y sus familias.

\section{DISCUSIÓN}

Dada nuestro tema inicial de investigación sobre descubrir la relación entre las elecciones ocupacionales, la salud y el bienestar bajo un análisis del sistema neoliberal, podemos decir que las elecciones ocupacionales se dan en un contexto social y cultural determinado; los participantes manifiestan elementos propios del sistema neoliberal en su discurso, tales como la competitividad, la acumulación de capital económico como prioridad, la supeditación de actividades productivas por sobre las recreativas que influyen en el cómo despliegan su ser ocupacional. En este sentido, las elecciones ocupacionales se encuentran restringidas a un contexto determinado, pudiendo ser contradictorias con interés y valores. Tales conceptos interrelacionados influyen en el ser ocupacional al momento de realizar una elección ocupacional, tal como actividad laboral, carrera a estudiar, pasatiempos y otros, lo que a su vez impacta en cómo se configura la participación ocupacional en las áreas productiva, autocuidado y de ocio y tiempo libre, evidenciándose la confrontación que sufren el sistema de valores con el de intereses en la supeditación de esta primera por el capital económico que entrega, que a su vez, constituye la vía que 
permite acceder a otros tipos de capitales, acceder a servicios y a ocupaciones de las áreas desplazadas.

Los objetivos de la investigación se han cumplido y la búsqueda de respuesta a las preguntas iniciales también; se ha indagado acerca de la influencia del sistema neoliberal en las elecciones ocupacionales de un grupo de en adultos económicamente activos de la ciudad de Valdivia; se han expuesto las elecciones ocupacionales manifestadas por estos, dando a conocer los elementos del sistema neoliberal que estos consideran como influyentes en la realización de elecciones ocupacionales, se han constatado otros factores que interactúan en esta interrelación y se ha analizado la relación que guardan con los conceptos de salud y la concepción de bienestar de los participantes.

En un principio, destacamos que desde las ciencias sociales existían diversos autores que hacen referencia explícita en como el sistema neoliberal repercute negativamente en la libertad de acción de los actores sociales, sin embargo desde las ciencias que estudia a los seres ocupacionales, se requiere incrementar el sustento teórico que permitiera ser un aporte al debate planteado por los críticos al neoliberalismo. Si bien esta investigación posee limitaciones que le son propias, quisiéramos que sea un llamado a la reflexión y análisis al momento de hablar sobre las elecciones ocupacionales y las decisiones que pueda tomar el ser humano como ser ocupacional y actor social, desde distintas disciplinas que permiten evidenciar la serie de factores que intervienen en tal proceso, y como esto puede repercutir en la práctica de la Terapia Ocupacional y ser un punto de vista para el debate planteado.

De los resultados, podemos afirmar que la lógica aspiracional presente, se contrapone con lo que describe Max-Neef (1993); el autor sostiene que existen nueve necesidades: subsistencia, protección, afecto, entendimiento, participación, ocio, creación, identidad y libertad y lo que logra satisfacerlas es lo que él denomina como satisfactores, los cuales varían de acuerdo al contexto del individuo (una persona puede acceder a educación universitaria o comprar un libro, satisfaciendo la misma necesidad de entendimiento). Sin embargo, la lógica aspiracional y la industria del marketing y el consumismo, presentan a tales satisfactores como una necesidad a cubrir; tal diferencia existente entre estos conceptos, no se reflejan en las respuestas de los participantes, evidenciando que los satisfactores son analizados como necesidades, pues el autor destaca su interrelación y no su homologación, influenciando así las elecciones ocupacionales, impactadas por la cultura del consumismo; la obtención del capital económico es el medio para obtener el bienestar social, lo que guarda relación con lo anteriormente planteado, generando así una retroalimentación positiva en el establecimiento de la necesidades que son realmente satisfactores. La no satisfacción de estas, provoca carencias que ponen en peligro el bienestar subjetivo, observándose que el capital económico impacta en la configuración de estos conceptos, donde una de las dificultades identificadas por los entrevistados tiene relación con la falta de capital económico (Bourdieu, 2003).

Si bien existe una reflexión sobre la posición que ocupan en el sistema socio-económicopolítico en el cual se encuentran, no se observan que sus acciones o elecciones sean ajenas al individualismo a pesar de manifestar una crítica hacia este, guardando relación con una 
de las estrategias del sistema como lo es la normalización de tal situación y sus consecuencias (Murillo, 2007).

Esta normalización señalada por los participantes sobre las desigualdades existentes y las características del sistema neoliberal y la normalización que tiene la sociedad al respecto de tal situación, coincide con los conceptos de poder de Foucault (2008) y de violencia simbólica de Bordieu (2003): la normalización constituye unos de los aspectos de la economía de poder, haciendo que el ejercicio de este sea difuso, y dificulta la identificación clara de quién es el dominante, pues no se puede responsabilizar a un individuo especifico como sostenedor, resultando así en la estrategias usada por el modelo neoliberal y su necesidad de auto sustentación; la resistencia a este poder es deslegitimizada, como lo son la criticada inequidad presente, categorizando de "utopías" o “efectos normales de la económica mundial". Los participantes destacan valores altruistas que están desapareciendo por el individualismo presente, señalando que les gustaría tener servicios públicos de calidad y con valor comunitario, valores que contiene el modelo económico planteado por Max-Neef (1993, sin embargo, este no tiene aceptación práctica en la economía global, siendo efectivas la estrategia de normalización y la deslegitimización.

Por ellos, ambos autores coinciden que debe atacarse el foco de estos ejercicios de poder, las instituciones e imponer nuevos arbitrarios, analizar las relaciones existentes y des-entregar el poder dado por la violencia simbólica existente, lo que nos gustaría como propósito ultimo de este trabajo de investigación: invitar a la reflexión desde la teoría planteada.

Sin embargo, dados los resultados obtenidos y su respectivo análisis y discusión, nos preguntamos ¿podemos realmente hablar de elecciones ocupacionales de forma libre y voluntaria cuando estas no son ajenas a la influencia de los elementos socio-políticoseconómicos-administrativos del sistema neoliberal? Considerando además sus respectivas estrategias de permanencia a través de la economía del poder que desarrolla Foucault (2008), afectando concretamente las dimensiones físicas del vivir o más específicamente el control de las poblaciones según Prudant (2014).

\section{Otra preguntas de investigación y limitaciones del estudio}

Esta investigación presenta limitaciones de acuerdo a la población entrevistada (económicamente activos de la ciudad de Valdivia, Chile), su objetivo no es de tipo cuantitativo, sino una visión cualitativa del fenómeno, la cual no pretende ser una generalización, sino más bien pretende entregar un análisis comprensivo de las narrativas des un foco retrospectivo.

Es de nuestra consideración que se levanten otras investigaciones que incorporen un foco prospectivo, por ejemplo con participantes que se encuentren finalizando la enseñanza media y que se vean enfrentados a hacer una elección ocupacional para su futuro, para analizar qué factores intervienen en esta elección. Otro aporte a la discusión, sería contar 
con investigaciones que utilicen un enfoque retrospectivo, pero en población económicamente pasiva, como adultos mayores o con población dedicada a actividades laborales no convencionales, para analizar qué factores fueron los influyentes en estas elecciones.

Además, consideramos que sería enriquecedor realizar una investigación con mayor profundidad acerca del análisis desde una perspectiva de género sobre los factores resultan más importantes al momento de realizar una elección ocupacional, desde el ámbito laboral como surgió en los análisis en el presente artículo, donde surgieron conceptos que se diferenciaron entre hombre y mujeres respecto a elementos de expresión y vocacionales en la actividad productiva; sería interesante ahondar sobre los roles que se adquieren en la adultez como el de madres o padres, y como se configura la expresión del ser a través de la ocupación haciendo la diferencia desde lo femenino y masculino.

Finalmente, creemos que es de suma importancia que las ciencias de la ocupación, la sociología, economía, sostenedores y creadores de políticas públicas dialoguen con el fin de tensar los conocimientos existentes y los factores que influyen en las elecciones y que hacer de las personas, con un debate amplio y que permita establecer los cambios que permitan una mayor libertad para los individuos, y así, la eliminación gradual de la subyugación de estos mediante estructuras de poder que violentamente actúan ante el conocimiento de muchos, y el silencio de otros: el rol profesional es justamente este, el utilizar los conocimientos necesarios en servicio de los actores sociales.

\section{REFERENCIAS BIBLIOGRÁFICAS}

Angelcos, N., Pérez, P., Sémbler, C. (2006). Los sectores medios ante la era neoliberal: Transformaciones y contradicciones del desarrollo en Chile. En Revista de sociología de Universidad de Chile, 20 (147-174). Recuperado de http://www.facso.uchile.cl/publicaciones/sociologia/articulos/20/2006AngelcosPerezySembler.pdf

Balasch, M., Montenegro, M. (2003). Una propuesta metodológica desde la epistemología de los conocimientos situados: las producciones narrativas. Recuperado de https://www.academia.edu/762651/Una_propuesta_metodol\%C3\%B3gica_desde_la_episte molog\%C3\%ADa_de_los_conocimientos_situados_Las_producciones_narrativas

Barrere, M. (s.f.). Problemas del derecho antidiscriminatorio: subordinación versus discriminación y acción positiva versus igualdad de oportunidades. Recuperado de http://www.uv.es/cefd/9/barrere3.pdf

Behm, H. (2011). Determinantes económicos y sociales de la mortalidad en América Latina. Salud Colectiva.7(2). Recuperado de http://www.scielo.org.ar/scielo.php?pid=S185182652011000200014\&script=sci_arttext 
Bissio, B. (1977). Sudáfrica: la crisis del apartheid. Nueva sociedad, 31(32), 231-240. Disponible en http://www.nuso.org/upload/articulos/355_1.pdf

Borón, A. (2008). Socialismo siglo XXI. ¿Hay vida después del neoliberalismo? (1a ed.). Buenos Aires, Argentina : Luxemburgo.

Bourdieu, P. (2003). Contrafuegos: reflexiones para servir a la resistencia contra la invasión neoliberal. (3a ed.). Barcelona, España. Editorial Anagrama.

Chomsky, N. (2004). El beneficio es lo que cuenta: Neoliberalismo y orden global. (4 ${ }^{\mathrm{a}}$ ed.). Critica. Barcelona.

Davis-Hamel, A. (2012). Successful neoliberalism?: State policy, poverty, and income inequality in Chile. International Social Science Review, 87(3/4), 79-101.

Fernandez, M. (1990). La cara oculta de la escuela: Educación y trabajo en el Capitalismo. Siglo Veintiuno Editores. Madrid.

Foucault, M. (2008). El Orden del discurso. Buenos Aires. Tusquets Editores.

Friedman, M. (1999). La economía monetarista. Gedisa. Barcelona.

Fromm, E. (1987). Anatomía de la destructividad humana. (11 a ed). Siglo XXI.

Gandarilla, J.(2005). America latina, en la conformación de la economía-mundo capitalista. Recuperado de: http://biblioteca.clacso.edu.ar/ar/libros/becas/critica/C02GSalgado.pdf

García, F. (2010). Aproximación critica a la crisis económica mundial: Sistema Capitalista, política Monetaria y globalización financiera. Pecvnia, 10, 75-94. Recuperado de: http://pecvnia.unileon.es/pecvnia10/10_075_094.pdf

Garcia, J. (2006). La tensión entre mérito e igualdad: el mérito como factor de exclusión.. Recuperado de http://www.tdx.cat/bitstream/handle/10803/9860/civico.pdf;jsessionid=8A6074CECC558E D91FAC09AC066F21C2.tdx2?sequence $=1$

Garreton, M.(2012).Neoliberalismo corregido y progresismo limitado. Chile: Editorial Arcis.

George, S. (1999) Una breve historia del neoliberalismo: veinte años de economía de élite y de oportunidades emergentes para el cambio estructural. Conferencia sobre Soberanía Económica, Bangkok, Tailandia. Recuperado el 29 de octubre de 2012 de http://www.france.attac.org/archives/spip.php/images/1/spip.php?article3225

Granados Toraño, R. (2002). Las reformas de los sistemas de salud: Tendencias Mundiales y Efectos en Latinoamérica y el Caribe. Revista Gerencia y Políticas en Salud. 1(3), 16-44.

Gunder, F. (1987). Capitalismo y subdesarrollo en América Latina. (9a ed.). Siglo Veintiuno Editores. México, D.F. 
Kielhofner, G. (2004). Modelo de Ocupación Humana. (3a ed.). Buenos Aires, Argentina. Editorial Panamericana.

Kronenberg, F., SimóAlgado S., Pollard, Nick. (2007) Terapia Ocupacional sin fronteras. Editorial Panamericana. Buenos Aires.

Marrero Lacería, V. (2006). Globalización neoliberal y su repercusión en las familias de los trabajadores. Revista Cubana de Salud y Trabajo, 7(1-2), 50-56.

Max-Neef, M. (1993). Desarrollo a Escala Humana: conceptos, aplicaciones y algunas reflexiones (1era Ed.). Montevideo, Uruguay: Editorial Nordan-Comunidad.

Meichsner, S. (2007). El campo político en la perspectiva teórica de Bourdieu. Iberoforum Voces $\quad y \quad$ Contextos, $3(2) . \quad$ doi: http://www.uia.mx/actividades/publicaciones/iberoforum/3/pdf/sylviam.pdf

Mondaca, A. (s.f.) Las falacias del neoliberalismo y la emergencia de los derechos humanos. Recuperado de http://www.rebelion.org/docs/86565.pdf

Moreno, Hugo (2006).Bourdieu, Fucoult y el poder. Voces y contextos. 2(1). Disponible en http://www.uia.mx/actividades/publicaciones/iberoforum/2/pdf/hugo_moreno.pdf

Murillo, S. (2007). Naturalizacion de la pobreza y de la desigualdad. Efectos políticos y subjetivos de las estrategias del Banco Mundial. Revista del CCC,1 (1) 1851-3263. Disponible en http://www.centrocultural.coop/revista/articulo/10/

Organización Mundial de la Salud OMS (1948). Subsanar las desigualdades de una generación. Comisión sobre determinantes en salud. Recuperado desde http://apps.who.int/iris/bitstream/10665/44084/1/9789243563701_spa.pdf

Oyarzún, N., Zolezzi, R., Núñez, J., Palacios, M. (2009). Hacia la construcción de las prácticas comunitarias de terapeutas ocupacionales en chile, desde una mirada socio histórica, desde 1972 hasta la actualidad, Revista Chilena de Terapia Ocupacional, 27 (9), $149-165$.

Prudant, S. (2014) Biopolítica del desarrollo y salud mental.

Rifkin, J. (2000) La era del acceso. Paidos. Buenos Aires.

Rodríguez, M., Carrillo, P. y Borrell, C. (2006).Desigualdades sociales en la salud, los estilos de vida y la utilización de servicios sanitarios en las comunidades autónomas. Observatorio de Salud de la Mujer, Agencia de Salud Pública de Barcelona. Recuperado de http://www.msc.es/organizacion/sns/planCalidadSNS/pdf/equidad/Desigualdades_sociales_ salud_y_SS.pdf

Rodríguez, F., Toledo, M., et al. (2000) La globalización y el modelo de Medicina General Integral en Cuba. Retos y oportunidades. Revista Cubana de Medicina General Integral, 16(1), 73-79. 
Soros, George (1999). El sistema capitalista global, en La crisis del capitalismo global: La sociedad abierta en peligro (pag 1). Plaza \& Janés, España.

Talavera Valverde, M. (2004, Octubre). La ocupación en las unidades de hospitalización psiquiátricas. Ponencia presentada en la II Jornada de Terapia Ocupacional en Salud Mental del País Vasco, Vitoria, España.

Toro, P.(28 de Noviembre del 2013). Estado subsidiario o benefactor ¿hacia donde transitamos? Pulso. Recuperado de http://www.pulso.cl/noticia/economia/economia/2013/11/7-34162-9-estado-subsidiario-obenefactor-hacia-donde-transitamos.shtml

Townsed, E., Brintnell, S., Staisey, N. (1990). Developing Guidelines for Client-Centred Occupational Therapy Practice. Canadian Journal of Occupational Therapy, 57(2), 69-76.

Vitale, L. (s.f.) Interpretación Marxista de la Historia en Chile. Recuperado de http://mazinger.sisib.uchile.cl/repositorio/lb/filosofia_y_humanidades/v20027291328vit ale05.pdf

Wallerstein, I. (2003) El capitalismo histórico. Siglo Veintiuno Editores. México D.F.

Western, M., Baxter, J., Pakulski, J., Tranter, B., Western, J., van Egmond, M., van Gellecum, Y. (2007). Neoliberalism, Inequality and Politics: The Changing Face of Australia. Australian Journal of Social Issues, 42(3), 401-418.

Wilcock, A. (1998). An Occupational Perspective of Health. Thorofare. Slack. 236 p.

Wilcock, A. (2006). An Occupational Perspective of Health. Thorofare. Slack. 260 p.

Zacarés, J. \& Serra, E.(1996) Creencias sobre la madurez psicológica y desarrollo adulto. Anales de Psicología, 12(1), 41-60, disponible en http://digitum.um.es/xmlui/bitstream/10201/10105/1/Creencias\%20sobre $\% 201 a \% 20$ madure z\%20psicologica\%20y\%20desarrollo\%20adulto.pdf 